\title{
Perceived effect on buoyancy of weight vs. gravitational force
}

\author{
DJ Wagner and Samuel Harryman \\ Department of Physics, Grove City College, 100 Campus Drive, Grove City, PA, 16127
}

As part of a larger investigation into students' conceptions about buoyancy, we investigated the prevalence of the belief that the gravitational force on an object changes when the object is placed in a fluid. We also investigated the effect of describing the force as "weight" instead of "gravitational force." During the first phase of the study (Winter 2016 to Fall 2016), students at two different institutions were asked to identify the correct free-body diagram (FBD) for a ball floating in water, and then for the same ball held down while fully submerged under water. Half of the students were shown FBDs involving "weight," and the other half were shown FBDs involving the "gravitational force." During the second phase of the study (Fall 2017 to Spring 2019), students at one of the institutions were asked to explicitly compare the strength of either the weight or the gravitational force on the ball when it was falling, floating, and held submerged. This paper will report on the fraction of students who indicated that the weight or gravitational force differed between the scenarios and will discuss the effects of both the question asked and the wording used to describe the force. 


\section{INTRODUCTION}

As part of the development of a standardized assessment for buoyancy, we have been cataloguing [1] and investigating the prevalence of [2-4] various buoyancyrelated conceptions. This paper focuses on the idea that the gravitational force on, or weight of, an object decreases when the object is placed in a fluid. We report on the prevalence of this conception in two United States college populations, and we discuss the effect on the results when the words "gravitational force of Earth on" are replaced with "weight of."

The idea that gravitational force and/or weight change

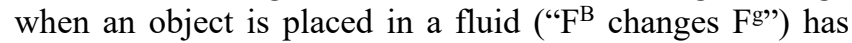
been identified in many populations [5-9], including our own interviews with US college students [10]. Additional studies [11-21] have discussed in depth the different meanings of the word "weight" in physics texts and instruction. Most physics texts present the "gravitational" definition of weight as the gravitational force on an object, either by the Earth or other nearest celestial object, or sometimes the net gravitational force. Yet other texts, and colloquial language, use an "operational" definition of weight - the scale reading or support force, or the $3^{\text {rd }}$ Law pair thereof, which will be influenced by non-inertial-frame effects and object motion.

Since the instructors of all courses in our study used the "gravitational" definition, we will not go further into the different definitions. We do, however, highly recommend the discussions in the references [13-16] to the instructor and/or author who wants to reflect on the challenges faced by students in assimilating the gravitational definition of weight. For simplicity we will abbreviate the force " $\mathrm{F}$ " throughout this paper.

The prior studies [11-21] of the influence of "weight" wording focus on falling or orbiting objects, with only the occasional mention of the connection to objects floating in water. Our study makes that connection, looking at the effect of word choice on the results of three different questions given to multiple populations. Below we describe our assessment protocol and populations, present the data showing the prevalence of the targeted conception in each population for each word choice, and then discuss what conclusions can be drawn from the data.

\section{METHODOLOGY AND QUESTIONS ASKED}

This paper analyzes responses to three questions from a larger assessment. Text of those questions is found in Fig. 1. In the first phase of the study, the free-body diagram (FBD) questions ("FBD floating" and "FBD submerged" [9]) were asked at both the University of Washington (UW), a large public research-one university, and Grove City College (GCC), a small private 4-year liberal arts college. In the second phase of the study, the third question (" $F$ g changes") was asked at GCC. Differing institutional norms required the assessment protocol to be different at each institution.
At UW, versions of the questions were included as part of online "pretests" administered over multiple quarters of the calculus-based sequence (UW Calc). These pretests were given before students had worked through a tutorial [22] on pressure. The lectures had covered pressure, but not buoyancy, so the assessment was "mid" instruction on fluids. The questions were in multiple-select format, often followed by a request for explanations of reasoning.

At GCC, the questions were included on two versions of a multiple-select bubble-sheet assessment given at the beginning and end of the semester in calculus-based (GCC Calc), trigonometry-based (GCC Trig), and conceptual courses (GCC Cncp). A few selected questions on the GCC assessment also ask for a short, written explanation, but the questions we discuss in this paper did not request explanations. We collected data on the " $\mathrm{F}^{\mathrm{g}}$ changes" question for two terms of each GCC class; since the students, textbook, and instructional approach to this topic in each term were similar for each course, we have combined the two terms for each course.

Two randomly distributed versions of the assessment were given to students in each class. In the questions discussed in this paper, one version of the assessment (vA) referred to "the gravitational force of the Earth on the ball," $\mathbf{F}_{\text {Earth on ball }}$ on FBDs, while the other version (vB) referred to the "weight of the ball," $\mathbf{W}_{\text {ball }}$ on FBDs. Note that the versions were randomly distributed both pre- and postinstruction at GCC, so the pre/post data is unmatched. We therefore can only make claims about the prevalence of conceptions after instruction and do not make any claims about the effects of specific instruction.

\section{RESULTS AND INTERPRETATION}

\section{A. Prevalence of targeted conception}

Figure 2 indicates the percentage of each population who indicated that the gravitational force was lower for an object in water than for the same object (at rest) in air. For the "FBD floating" question, this includes students who chose any of the options with $\mathrm{F}^{\mathrm{g}}$ less than the out-of-water value of two units: option A, C, E, or G. Similarly for the "FBD submerged" question: anyone choosing option $\mathrm{C}, \mathrm{D}, \mathrm{E}$, or $\mathrm{H}$ is included on the graph. The " $\mathrm{F}$ changes" question is more challenging to analyze, with a large number of answer permutations chosen by students due to the multiple-select format. We chose to count any combination of options B, C, $\mathrm{E}$, and/or F, with or without A, D, and/or G, as indicating that being in the water changes the gravitational force.

Across all populations, questions, and versions, the fraction of students indicating that $\mathrm{F}^{\mathrm{g}}$ changes in water varied between 9\% ("Fg changes" GCC Calc S18/S19 vA Post) and $54 \%$ ("FBD submerged" GCC Cncp F16 vB Pre). Thus the idea that buoyancy affects the gravitational force is widespread, persisting through instruction. (This study did not look at the impact of specific instruction, and instruction 


\section{"FBD Floating"}

Which of the following force ("free body") diagrams corresponds to the ball floating motionless in water (without being pushed by the hand)? Forces are to scale, and the gravitational force of the Earth on the ball out of water corresponds to 2 units on the graph.

A
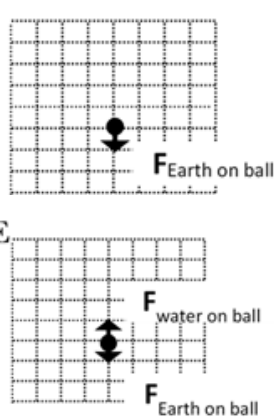

B

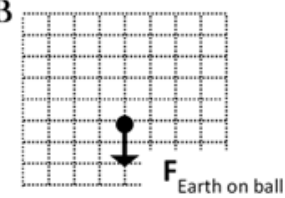

F

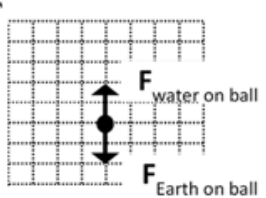

C

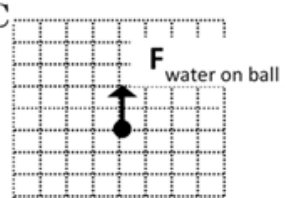

G

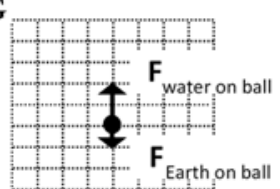

$\mathrm{D}$
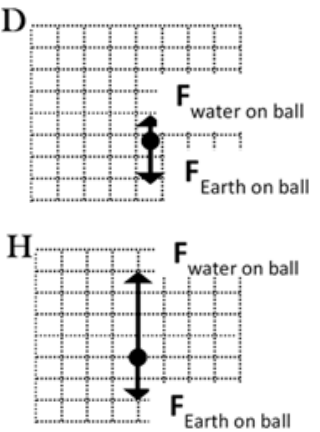

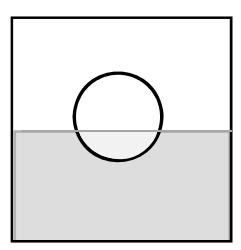

I. None of above

"FBD Submerged"

Which of the following force ("free body") diagrams corresponds to the ball at rest and held completely submerged in water? Forces are to scale, and the gravitational force of the Earth on the ball out of water corresponds to 2 units on the graph.

A

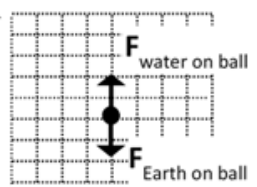

$\mathrm{E}_{\mathrm{P}}$

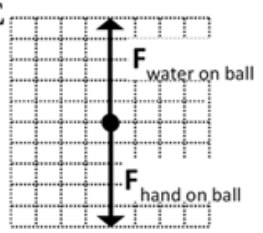

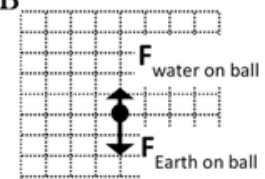

F

$\mathbf{F}$

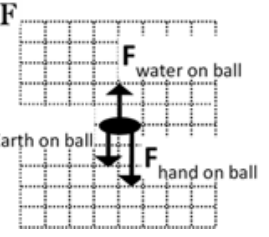

$\mathrm{C}$

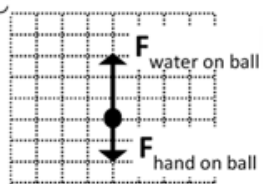

G

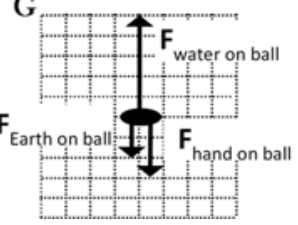

D

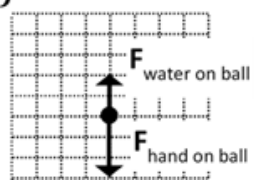

H

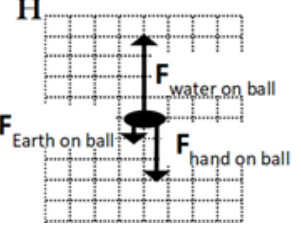

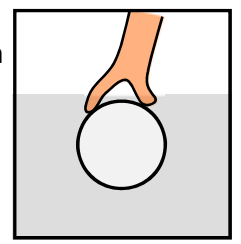

I. None of above

\section{"Fg changes"}

The three figures displayed show a ball which falls through air (Fig A), ends up floating in water (Fig B), and then is held motionless underwater by a hand (Fig C). Which of the following statements about the gravitational force on the ball is/are possible? Select all that apply.

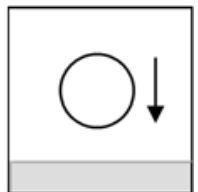

Fig A

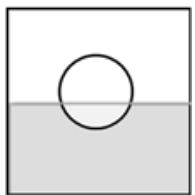

Fig B

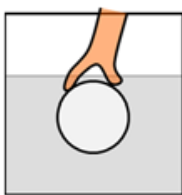

Fig C

A. The gravitational force on the falling ball (Fig A) is less than the gravitational force on the ball when it is at rest on the ground.

B. The gravitational force on the floating ball (Fig B) is less than the gravitational force on the ball when it is at rest on the ground.

C. The gravitational force on the submerged ball (Fig C) is less than the gravitational force on the ball when it is at rest on the ground.

D. The gravitational force on the falling ball (Fig A) is zero.

E. The gravitational force on the floating ball (Fig B) is zero.

F. The gravitational force on the submerged ball (Fig C) is zero.

G. The gravitational force on the ball is the same in all three figures.

H. None of the above.

FIG. 1. The three questions featured in this paper, with the wording from version A. Version B had the same questions, with "the gravitational force [of the Earth] on" replaced with "the weight of," and the FBD symbol $\mathbf{F}_{\text {Earth on ball }}$ replaced with $\mathbf{W}_{\text {ball. }}$ 
was not designed to address this specific conception. We merely notice that a non-negligible fraction of students evinces this conception post-instruction in each population.)

The data in Fig. 2 are messy, as one would expect for a multiple-question assessment given multiple times in multiple courses with multiple instructors and audiences. The prevalence of the conception does seem to be higher for vB ("weight" wording) in most course/time data sets, but there are exceptions.

To test for statistical significance, we performed a $3 \times 2 \times 2 \times 3$ between subjects ANOVA on the GCC data, with the conception as the dependent variable, and course (3), version (2), pre/post offering (2), and question (3) as the fixed factors. This showed an overall statistically significant dependence on version $(p=0.003)$. (We did not include the UW data in this analysis, since there was not pre/post data for UW). The 4-way interaction was significant $(p=0.025)$, confirming that each course/time/question data set did not show the same trend, even though there was an overall dependence on version.

We also performed a $3 \times 2 \times 2$ test for each question, finding that version had a significant effect only for "FBD submerged." ( $p=0.027$ for "FBD submerged, $p=0.107$ for "FBD floating,", and $p=0.287$ for " $\mathrm{F}^{\mathrm{g}}$ changes".) Because the GCC Cncp data exhibits a different $\mathrm{vA} / \mathrm{vB}$ trend from the other two courses for the " $F^{g}$ changes" question, we re-ran the analysis without the Cncp data. The " $\mathrm{F}$ ' changes" data for the
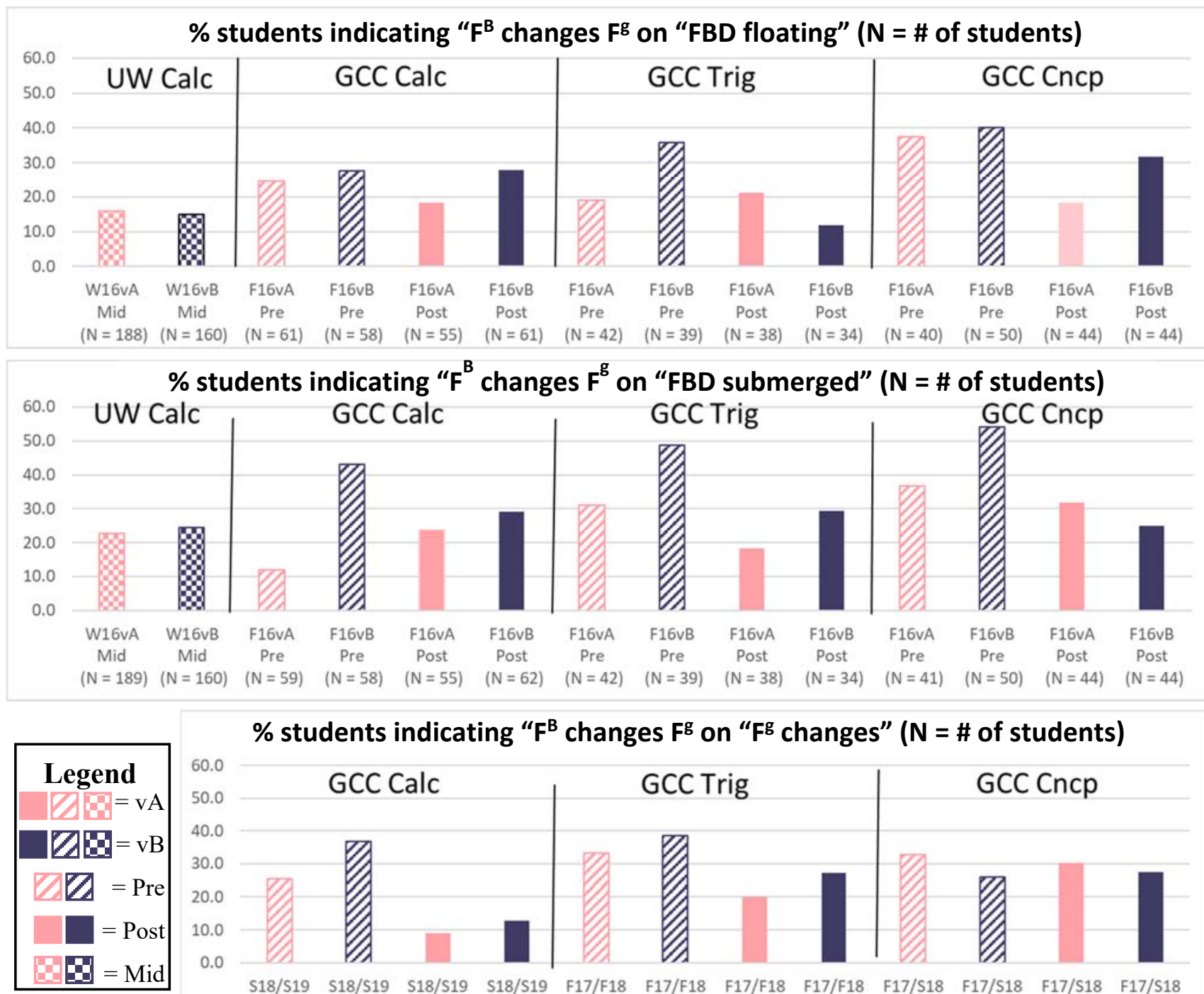

\% students indicating " $\mathrm{F}^{\mathrm{B}}$ changes $\mathrm{F}^{\mathrm{g}}$ on " $\mathrm{F}^{\mathrm{g}}$ changes" ( $\mathrm{N}=$ \# of students)

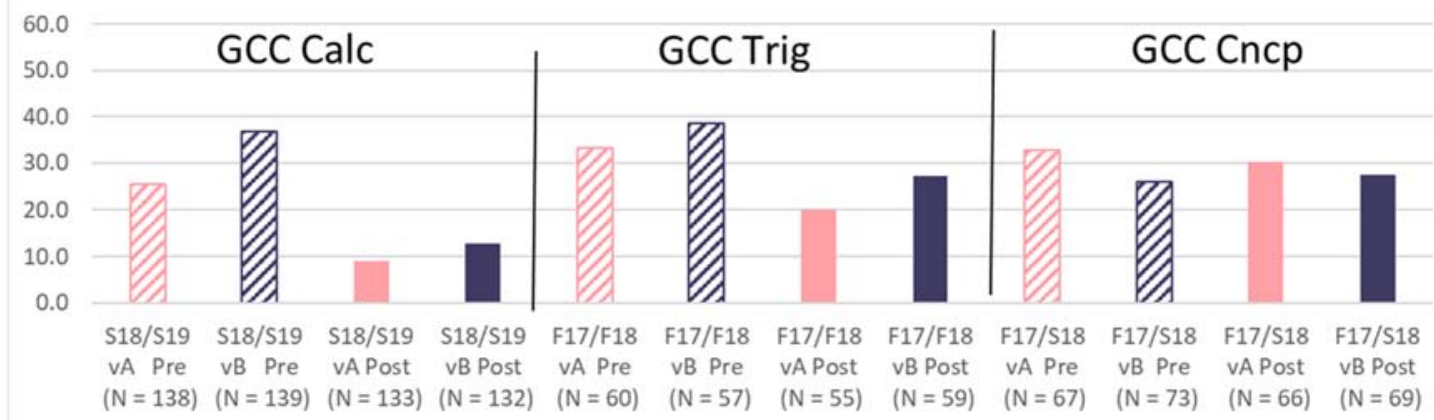

FIG. 2. The percentage of each sample selection options consistent with the " $\mathrm{F}^{\mathrm{B}}$ changes $\mathrm{F}^{\mathrm{g}}$ " conception, for each of the three targeted questions. (Option A, C, E, or G for FBD Floating, Option C, D, E, or H for FBD Submerged, and any combination of B, $\mathrm{C}, \mathrm{E}$, and/or $\mathrm{F}$ (with or without $\mathrm{A}, \mathrm{D}$, and/or $\mathrm{G}$ ) for $\mathrm{F}^{\mathrm{g}}$ changes. vA uses the "gravitational force" wording, while vB uses "weight." Term labels use $\mathrm{W}=$ winter quarter, $\mathrm{F}=$ fall semester, and $\mathrm{S}=$ spring semester, with $16=2016$, etc. 
remaining two courses is significantly affected by the version $(p=0.035)$.

\section{CONCLUSIONS}

Many other studies have shown that students often claim that gravitational force decreases significantly, or even goes to zero, in freefall situations of perceived "weightlessness." We have shown that the same difficulties arise in situations involving buoyancy, even when the relevant force is clearly labeled as gravitational and not as the ambiguous term "weight." The prevalence of these difficulties understandably varies by population, but the conception is evinced by a non-negligible fraction of students at two very different institutions, and at three different courses at one of those institutions, in answering different questions.

Often, students' belief that $\mathrm{F}^{\mathrm{g}}$ decreases when in an orbiting satellite is attributed at least in part to misunderstanding the effect of scale on the distancedependence of $\mathrm{F}^{\mathrm{g}}$, or possibly the conflation of gravity and air pressure $[12,14,16,18,19]$. No such cause can be attributed to our data, indicating that the belief in a varying $\mathrm{F}^{\mathrm{g}}$ involves more underlying reasonings than previously reported.

Although the trend was not uniform for all our populations, students in our study were overall more likely to evince the " $\mathrm{F}^{\mathrm{B}}$ changes $\mathrm{F}^{\mathrm{g}}$ " conception if the word "weight" were used instead of "gravitational force." This trend appears even though all instruction defined $\mathrm{F}^{\mathrm{g}}$ and weight to be synonymous. We find it interesting that, while the conception was present in the UW population, the UW data show no dependence on version. Similarly, the GCC Cncp data for " $F$ g changes" shows no version dependence. The authors do not have an explanation for why these two data sets should be less version-dependent than the others, when all four courses used the gravitational definition of weight. A more targeted study using matched pre/post data would be necessary to draw concrete conclusions.

Multiple studies [16-19] suggest that instruction using the operational definition of weight can avoid many common difficulties. We did not perform a randomized study of the difference of instruction in our context, but the GCC Cncp course in fall 2018 was incidentally taught using the operational definition of weight. According to this definition, weight does change, but $\mathrm{F}^{\mathrm{g}}$ does not, when an object is placed in water; the results in Fig. 3 show the resulting large dependence upon assessment version postinstruction. It is interesting to note that the fall $2018 \mathrm{vA} \%$ correct post-instruction was $83 \%$, indicating that these students taught with the operational definition of weight well understood that $\mathrm{F}^{\mathrm{g}}$ does not change in water. (The highest of any previous GCC Cncp course was 74\% post-instruction vA in fall 2017). Since instructor, text, and content coverage

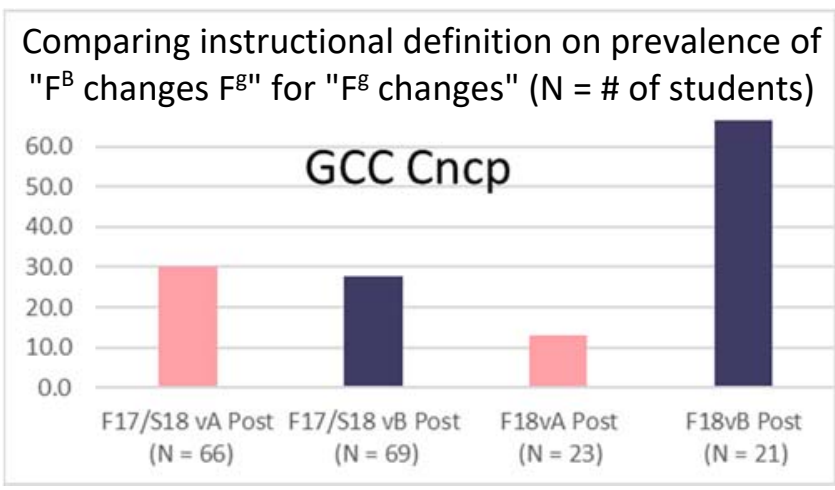

FIG.3. Post-instruction results for GCC Cncp on " $\mathrm{F}^{\mathrm{g}}$ changes" question. F17/S18 instruction used the gravitational definition of weight, while F18 instruction used the operational definition.

were all different for the two offerings, we cannot claim causality, but the results do suggest value to the recommendations made in references [16-19]. Again, this is an interesting issue requiring more targeted future study.

This research confirms the findings of freefall studies that students do not adequately grasp the relative constancy of $\mathrm{F}^{\mathrm{g}}$ at or near the surface of Earth. Confusion over the meaning of the word "weight" undoubtedly contributes to this difficulty. Instructors cannot easily change how "weight" is defined in their courses, since using notation and language different than that of the chosen textbook provides a different set of hurdles to learning. As a first step, the authors plan to follow advice given in references [20,21,23,24] and avoid the use of the word "weight" entirely in the assessment under development. While use of common language is desirable in concept inventories, the ambiguity of the common word "weight" makes its usage undesirable. Until textbook authors take note of the research on this subject and change the language in the texts, the best an instructor can do is to do likewise and avoid "weight" as much as possible. As pointed out in references [20,21], this is best paired with a class discussion of the controversy and different meanings of the term. This study, and others, have shown the inadequacy of simply stating the chosen definition and assuming students will use that definition going forward.

\section{ACKNOWLEDGMENTS}

The authors would like to thank Dr. Gary Welton of GCC for his invaluable help with statistical analysis and interpretation. We wish to thank Dr. Peter Shaffer and the entire UW PEG group for support, access to students, and assistance. We also wish to thank the following student members of the GCC PER and UW PEG groups who contributed to this project: Shannon Armstrong, Sheh Lit Chang, Kristen Hephner, Mercedes Mansfield, and Zackary Spang. 
[1] D. Wagner, "A Taxonomy of Conceptions about Buoyancy," poster PST1D35 presented at 2016 Summer meeting of American Association of Physics Teachers; paper in preparation.

[2] D. Wagner, S. Cohen, and A. Moyer, "Addressing Student Difficulties with Buoyancy," in 2009 Physics Education Research Conference Proceedings, edited by M. Sabella, C. Henderson, and C. Singh (Ann Arbor, MI, 2009), $<$ https://www.compadre.org/Repository/document/ServeFile. $\mathrm{cfm}$ ?ID=9507\&DocID=1382>.

[3] D. Wagner, E. Carbone, and A. Lindow, "Exploring Student Difficulties with Buoyancy," in 2013 Physics Education Research Conference Proceedings, edited by $\mathrm{P}$. V. Engelhardt, A. D. Churukian, and D. L. Jones (Portland, OR, 2013). <https://www.compadre.org/PER/document/ ServeFile.cfm? ID $=13152 \&$ DocID $=3699>$

[4] D. Wagner and P. Shaffer, "The Prevalence of Selected Buoyancy Alternate Conceptions at Two Colleges," in 2017 Physics Education Research Conference Proceedings, edited by L. Ding, A. L. Traxler, and Y. Cau (Cincinnati, OH, 2017,) $<$ https://www.compadre.org/Repository/document/ServeFile. $\mathrm{cfm}$ ?ID $=14658 \&$ DocID $=4835>$.

[5] F.F. Camacho and L.G. Cazares, "Partial Possible Models: An Approach to Interpret Students' Physical Representation," Sci. Educ. 82, 15-29 (1998).

[6] M.G. Hewson, "The acquisition of scientific knowledge: Analysis and representation of student conceptions concerning density." Sci. Educ. 70, 159 (1986).

[7] T.G. Moore and A.G. Harrison, "Floating and sinking: everyday science in middle school" (2004). Retrieved July 5, 2014 from http://www.aare.edu.au/data/publications/2004/ moo04323.pdf.

[8] A. Zeineddin and A. Fouad, "On coordinating theory with evidence: The role of epistemic commitments in scientific reasoning among college students," Eurasion J. Math., Sci. and Technol. Educ. 4, 153 (2008).

[9] James Vesenka, private communication. Our free-body diagram questions are motivated by questions developed by James Vesenka.

[10] This conception was expressed by multiple subjects in preinstruction interviews of GCC students carried out by the GCC PER group in fall, 2013 and spring, 2014.

[11]R.C. Morrison, "Weight and Gravity - the Need for Consistent Definitions," Phys. Teach. 37, 51 (1999).

[12] G. Tural, A.R. Akdeniz, and N. Alev, "Effect of 5E Teaching Model on Student Teachers' Understanding of Weightlessness," J. Sci. Educ. and Technol. 19, 470 (2010).

[13] U. Besson, "Do things weigh more or less in the mountains?" Phys. Educ. 41, 391 (2006).

[14] I. Galili, "Interpretation of Students' Understanding of the Concept of Weightlessness," Res. Sci. Educ. 25, 51 (1995).

[15] R. Taibu, D. Rudge, and D. Schuster, "Textbook presentations of weight: Conceptual difficulties and language ambiguities," Phys. Rev. ST - PER 110100117 (2015).

[16]I. Galili, V. Barm, and Y. Brosh, "Teaching Weight-Gravity and Gravitation in Middle School - Testing a New Instructional Approach," Sci. \& Educ. 25, 977 (2016).

[17] V. Bar, Y. Brosh, and C. Sneider, "Weight, Mass, and Gravity: Threshold Concepts in Learning Science," Scic. Educ. 25, 22 (2016).

[18] I. Galili, "Weight versus Gravitational Force: Historical and Educational Perspectives," Int. J. Sci. Educ. 23, 1073 (2001).
[19] I. Galili and D. Kaplan, "Students' Operations with the Weight Concept," Scic. Educ. 80, 457 (1996).

[20] R. Taibu, “Terms vs. Concepts - The Case of Weight,” Phys. Teach. 55, 34 (2017)

[21] R. Taibu, D. Schuster, and D. Rudge, "Teaching weight to explicitly address language ambiguities and conceptual difficulties," Phys. Rev. ST - PER 13010130 (2017).

[22]L. C. McDermott, P. S. Shaffer, and the Physics Education Group at the University of Washington, Tutorials in Introductory Physics, First Edition, Prentice Hall, Upper Saddle River, NJ, 2002.

[23] R. Brown, "Weight - Don't Use the Word at All," Phys. Teach. 37, 241 (1999).

[24] A. Louro, “Banish 'weight'?” Phys. Teach. 49, 196 (2011). 